\title{
Joint analysis of left ventricular expression and circulating plasma levels of Omentin after myocardial ischemia
}

\author{
Louis A. Saddic', Sarah M. Nicoloro², Olga T. Gupta ${ }^{3}$, Michael P. Czech $^{4}$, Joshua Gorham ${ }^{5}$, Stanton K. Shernan ${ }^{6}$, \\ Christine E. Seidman7, Jon G. Seidman ${ }^{5}$, Sary F. Aranki ${ }^{8}$, Simon C. Body ${ }^{6}$, Timothy P. Fitzgibbons ${ }^{9 \dagger}$ \\ and Jochen D. Muehlschlegel ${ }^{6^{*}+}$ (1)
}

\begin{abstract}
Background: Omentin-1, also known as Intelectin-1 (ITLN1), is an adipokine with plasma levels associated with diabetes, obesity, and coronary artery disease. Recent studies suggest that ITLN1 can mitigate myocardial ischemic injury but the expression of ITLN1 in the heart itself has not been well characterized. The purpose of this study is to discern the relationship between the expression pattern of ITLN1 RNA in the human heart and the level of circulating ITLN1 protein in plasma from the same patients following myocardial ischemia.
\end{abstract}

Methods: A large cohort of patients $(n=140)$ undergoing elective cardiac surgery for aortic valve replacement were enrolled in this study. Plasma and left ventricular biopsy samples were taken at the beginning of cardiopulmonary bypass and after an average of $82 \mathrm{~min}$ of ischemic cross clamp time. The localization of ITLN1 in epicardial adipose tissue (EAT) was also further characterized with immunoassays and cell fate transition studies.

Results: mRNA expression of ITLN1 decreases in left ventricular tissue after acute ischemia in human patients (mean difference 280.48, $p=0.001$ ) whereas plasma protein levels of ITLN1 increase (mean difference 5.24, $p<0.001$ ). Immunohistochemistry localized ITLN1 to the mesothelium or visceral pericardium of EAT. Epithelial to mesenchymal transition in mesothelial cells leads to a downregulation of ITLN1 expression.

Conclusions: Myocardial injury leads to a decrease in ITLN1 expression in the heart and a corresponding increase in plasma levels. These changes may in part be due to an epithelial to mesenchymal transition of the cells that express ITLN1 following ischemia.

Trial Registration Clinicaltrials.gov ID: NCT00985049

Keywords: Ischemia, Adipokine, Omentin, RNA-seq, Cardiovascular

\section{Introduction}

Adipose tissue is a complex organ whose functions extend well beyond its canonical role in energy storage [1-3]. Adipose tissue can act as an endocrine organ

\footnotetext{
*Correspondence: jmuehlschlegel@partners.org

${ }^{\dagger}$ Timothy P. Fitzgibbons and Jochen D. Muehlschlegel contributed equally to this work

${ }^{6}$ Department of Anesthesiology, Perioperative and Pain Medicine, Brigham and Women's Hospital, Harvard Medical School, CWN L1, 75 Francis Street, Boston, MA 02115, USA

Full list of author information is available at the end of the article
}

through the secretion of adipokines which have the ability to act locally and remotely. Adipokines have diverse roles including the regulation of angiogenesis, metabolism, inflammation, and cell survival/death in many tissue types. Recently, investigators have begun to uncover the pivotal role these secreted substances have in many pathological processes including cardiovascular disease [4-6].

Omentin-1, also know as Intelectin-1 (ITLN1), is an adipokine that in humans is primarily expressed in visceral adipose tissue (VAT), with very little expression in subcutaneous adipose tissue (SAT) [7]. More specifically, 
the vast majority of ITLN1 expression from adipose tissue is derived from the stromal vascular compartment with only trace amounts produced in adipocytes [8]. This is in contrast to many other adipokines which are primarily expressed in fat cells [7]. In humans, ITLN1 can be detected in the circulation, and the level of this circulating pool of protein has been correlated with many disease processes. For example, the plasma concentration of ITLN1 is reduced in patients with obesity and diabetes $[9,10]$. With respect to cardiovascular disease, ITLN1 is decreased in metabolic syndrome patients with carotid atherosclerosis [11] along with patients with coronary artery disease (CAD) [12, 13]. Functionally, ITLN1 has been shown to reduce TNF-induced vascular inflammation in human endothelial cells [14]. In rats, ITLN1 promotes vasodilation in blood vessels [15] and protects against cerebral ischemia by promoting angiogenesis and inhibiting apoptosis through stimulating AKT and endothelial nitric oxide synthase [16]. A recent study also showed that systemic administration of human ITLN1 in mice leads to a reduction in infarct size and myocyte apoptosis after ischemia/reperfusion injury through phosphorylation of AMPK and AKT [17]. These protective properties of ITLN1 are further substantiated by other studies which have shown that increasing levels of plasma ITLN1 in humans correlate with higher myocardial index scores [17] and a lower frequency of ischemic EKG changes [18].

Despite these preliminary studies, the expression of this cardioprotective adipokine in the heart has not been well characterized. Studies have shown that the epicardial fat, a special type of visceral fat, expresses high amounts of ITLN1 and this expression may be related to underlying CAD $[19,20]$. Early studies on the global expression of ITLN1 demonstrated that this adipokine has low levels of expression in other organs including the heart [7] but further studies in humans have been limited given the difficulty of obtaining human heart tissue. Therefore, we sought to determine the effects of myocardial ischemia on the expression of ITLN1 in the human left ventricle and circulating levels of ITLN1 protein in a large population of patients.

\section{Methods}

\section{Human subjects and left ventricular samples}

Patients presenting for elective aortic valve replacement surgery by a single surgeon were enrolled in this study. They provided written informed consent with Institutional Review Board approval. Clinical characteristics of the study population are shown in Table 1. Patients were deemed to have CAD if obstructive lesions were noted on left heart catheterization just prior to aortic valve surgery requiring concomitant revascularization of at least
Table 1 Clinical characteristics of study patients

\begin{tabular}{ll}
\hline Characteristics & Statistic max $\mathbf{n}=\mathbf{1 4 0}$ \\
\hline Gender (male) & $86(61.43 \%)$ \\
Age & $71.24 \pm 12.09$ \\
Degree of aortic stenosis & \\
None to trivial & $10(7.14 \%)$ \\
Mild & $0(0 \%)$ \\
Moderate & $15(10.71 \%)$ \\
Severe & $115(82.14 \%)$ \\
Degree of aortic insufficiency & \\
None to trivial & $61(43.57 \%)$ \\
Mild & $38(27.14 \%)$ \\
Moderate & $25(17.86 \%)$ \\
Severe & $16(11.43 \%)$ \\
LV EF (\%) & $57.36 \pm 10.97$ \\
AX time (min) & $81.73 \pm 27.89$ \\
BMI (kg/m²) & $30.76 \pm 7.13$ \\
CAD (\%) & $67(47.86 \%)$ \\
HgA1C (\%) & $6.08 \pm 0.90$ \\
Day 1 CKMB (mcg/L) & $32.00 \pm 24.57$ \\
Pre ITLN1 (FPKM) & 59.96 IQR 5.43-303.57 $\mathrm{n}=132$ \\
Post ITLN1 (FPKM) & 2.46 IQR 0.84-11.00 $\mathrm{n}=132$ \\
Pre ITLN1 (ng/ml) & $32.23 \pm 15.22 \mathrm{n}=106$ \\
Post ITLN1 (ng/ml) & $37.46 \pm 16.84 \mathrm{n}=106$ \\
\hline
\end{tabular}

Statistics are mean values and standard deviations for continuous variables except ITLN1 mRNA levels which are described with median and interquartile range. Statistics for categorical variables are number of patients and percentage of patients

LVEF left ventricular ejection fraction, $A X$ time aortic cross clamp time, $B M I$ body mass index, $C A D$ coronary artery disease, HgA1c hemoglobin $A 1 C, C K M B$ creatine kinase MB fraction, IQR interquartile range

one vessel. Punch biopsies ( $3-5 \mu$ g total RNA content) were obtained intra-operatively from the site of a routinely placed surgical vent in the anterolateral apical left ventricle at the initiation of cardiopulmonary bypass $(\mathrm{CPB})$ and again after an average of $81.73 \pm 27.89 \mathrm{~min}$ of aortic cross clamp time. Specifically, the biopsies were taken from within the myocardium and did not include infarcted tissue. Pre-ischemia and post-ischemia biopsies were taken from the same site in the track made for the LV vent. During aortic cross clamping, the heart was infused with intermittent cold blood cardioplegia (8:1 blood to crystalloid ratio) for myocardial protection.

\section{RNA sequencing}

Tissue samples were immediately placed in RNAlater ${ }^{\circledR}$ (Ambion, Life Technologies, USA), and after $48 \mathrm{~h}$ at $+4{ }^{\circ} \mathrm{C}$ were stored at $-80{ }^{\circ} \mathrm{C}$ until RNA extraction. Total RNA was isolated with Trizol and RNA quality was assessed using the Agilent Bioanalyzer 2100 (Agilent) with no samples being excluded for poor quality. Library preparation and sequencing has been 
described previously [21], but briefly, ribosomal RNA was removed by performing 1-2 washings of RNA annealed to poly-T oligos beads (Invitrogen). RNAs were reverse transcribed using random hexamers (Invitrogen). Double-stranded DNA (dsDNA) synthesis was performed using Pol I and RNA-ase H. Short fragments were purified with QiaQuick PCR extraction kit (Qiagen) and resolved with EB buffer for end reparation and poly(A) addition then ligated with sequencing adaptors for cluster generation and sequencing on the Illumina HiSeq 2000 (Illumina, San Diego, CA). As samples were analyzed at different times, different read lengths were employed, initially using single-end reads and then transitioning to paired-end reads ranging from 36 to 100 base pairs.

\section{Read alignment, transcript quantification, and differential gene expression analysis}

Raw reads produced by the Illumina sequencer imaging files were filtered to remove reads containing adaptor sequences, containing $>5 \%$ unknown nucleotides, or having $>50 \%$ of reads with base quality scores $<5$. Using Tophat v2.0.5 and Bowtie2 [22-24], reads were aligned to the Homo Sapiens reference genome (UCSC hg19). For paired-end reads, mate inner distance was set at $165 \mathrm{bp}$ and mate standard deviation at $37 \mathrm{bp}$. For other parameters, we used the default settings in Tophat v2.0.5 and Bowtie2. Final read alignments having more than two mismatches or more than two gaps or more than two edit distances were discarded. The anchor length was 8 without any mismatches being tolerated. The minimum and maximum intron lengths were 70 and 500,000 respectively. The maximum insertion and deletion lengths were 3. Fragments per kilobase per million mapped reads (FPKM) levels were determined using Cufflinks [25]. Differential expression analysis was performed using Limma (v3.30.11) [26]. A linear model was constructed to test the effect of ischemia on gene expression, denoted $y$, while blocking on the patient from which each sample was collected, which adjusts for within and betweensample variability:

$$
\mathrm{y} \sim \beta_{1} \text { (patient) }+\beta_{2} \text { (ischemia) }
$$

where $\beta$ denotes regression coefficients. Read counts were normalized while incorporating sample-level weights using voom [27], and genes were filtered for inclusion in differential expression analysis in accordance with practices established by the authors of edgeR [28]. For each gene, positive or negative log2fold change, which was interpreted as an increase or decrease, respectively, in gene expression due to ischemia, was determined. $p$-values were adjusted for multiple comparison testing using the Benjamini Hochberg method.

\section{Epicardial, visceral, and subcutaneous fat samples}

Human visceral (omental) and abdominal subcutaneous adipose tissue samples were collected from patients undergoing weight loss reduction surgery at the University of Massachusetts Medical School between 2005 and 2009 [29]. The microarray data from visceral (VAT) and subcutaneous adipose tissue (SAT) samples have been deposited in the GEO database under accession code GSE20950. Samples of epicardial adipose tissue (EAT) adjacent to the right coronary artery, and SAT from the sternum, were collected from patients without CAD who were having elective surgery for valvular heart disease. RNA was isolated using QIAGEN mini-lipid RNA extraction kits and analyzed on an Agilent 2100 Bioanalyzer. Complementary RNA (cRNA) synthesis and hybridization to Affymetrix 1.0 ST microarrays was performed by the UMASS Medical School Genomics Core Facility. For both studies, informed consent was given by the patients and the study was approved by the University of Massachusetts Medical School Institutional Review Board. Subcutaneous (inguinal) and visceral (gonadal) adipose tissue was harvested from 13 week old male C57BL/6 mice fed normal chow. RNA from paired samples $(n=6)$ was prepared with QIAGEN mini-lipid RNA extraction kits and analyzed on an Agilent 2100 Bioanalyzer. Complementary RNA (cRNA) synthesis and hybridization to Affymetrix Mouse Gene 1.0 ST microarrays was performed by the UMASS Medical School Genomics Core Facility. This data is publically available in the GEO database under accession code GSE28440.

\section{ITLN1 ELISA}

Plasma samples were obtained from venous blood obtained before and after $\mathrm{CPB}$ in the same population of cardiac surgical patients from which the left ventricular biopsies were taken. Concentrations of ITLN1 were measured using ELISA (Millipore \#EZHOMNTN1-29K) according to the manufacturer's instructions.

\section{ITLN1 Immunohistochemistry}

Sections of EAT were fixed overnight in 10\% formalin, sectioned, and stained for ITLN1 using a sheep antihuman antibody (R\&D Biosystems \#AF4254) at $10 \mu \mathrm{g} / \mathrm{ml}$. The secondary antibody was a donkey Anti-Sheep IgG NorthernLights NL557-conjugated antibody. Sectioning and immunofixation were done by the UMASS Morphology core.

\section{Mesothelial cell culture and qRT-PCR}

Primary human mesothelial cells were purchased from Zen-Bio. Cells were cultured according to the manufacturer's instructions (Human Adult Mesothelial Cell Manual ZBM0025.01). Briefly, cells were thawed and plated in 
MSO-1 media at a density of 5000 cells $/ \mathrm{cm}^{2}$. After they were $85-90 \%$ confluent, they were split at a 1:6 ratio and seeded onto collagen coated plates. On day 4, cells were serum starved for $24 \mathrm{~h}$ with Media 199 (Gibco) containing $5 \%$ penicillin/streptomycin, normocin, and $0.5 \%$ FBS. After $24 \mathrm{~h}$, the media was changed and vehicle or $5 \mathrm{ng} / \mathrm{ml}$ TGF $\beta 1$ (Sigma T7039) was added. Cells were harvested for RNA extraction after $48 \mathrm{~h}$ of TGF $\beta 1$ treatment. RNA was isolated using TRIzol as previously described. cDNA was synthesized with the iScript cDNA systhesis kit (BioRad) with $1 \mu \mathrm{g}$ RNA template. qRT-PCR was performed using the iTaq Universal Sybergreen supermix (BioRad) and a CFX96 Connect Real Time Detection System. Relative expression was normalized to ribosomal phosphoprotein P0 (RPLP0) using the method of Livak et al. [30]. Primer sequences were obtained from primer bank and were as follows: E-cadherin (CDH-1) (5'cgagagctacacgttcacgg $3^{\prime}$, $3^{\prime}$ gggtgtcgagggaaaaatagg $\left.5^{\prime}\right)$; VMAC (5'gccctagacgaactgggtc $3^{\prime}, \quad 3^{\prime}$ ggctgcaactgcctaatgag $\left.5^{\prime}\right) ;$ fibronectin $\left(5^{\prime}\right.$ 'ggtggctgtcagtcaaag3', 3'aaacctcggcttcctccataa5'); SNAI1 (5' tcggaagcctaactacagcga $3^{\prime}$, 3'agatgagcattggcagcgag $5^{\prime}$ ); SNAI2 (5'cgaactggacacacatacagtg $3^{\prime}, 3^{\prime}$ ctgaggatctctggttgtggt $5^{\prime}$ ).

\section{Statistical analysis}

Baseline continuous variable patient characteristics are presented as means and standard deviations except ITLN1 mRNA levels which are described with median and interquartile range. Categorical variables are described with total number and percentages. Differences between pre-ischemia and post-ischemia ITLN1 protein levels and ITLN1 RNA levels were determined using a paired $t$ test. Univariate linear regression analysis was performed on pre-ischemia ITLN1 RNA and ITLN1 plasma levels against each other and clinical parameters including age, body mass index (BMI), hemoglobin A1c (HgA1c), sex, CAD, and post-operative day 1 creatine kinase $\mathrm{MB}$ fraction (CKMB). Similarly, univariate linear regression analysis was also conducted on post-ischemia ITLN1 RNA and ITLN1 plasma levels against the same clinical parameters in addition to aortic cross clamp time. These studies were conducted in R studio [31] using the general linear model function and results are reported as $p$-values and beta coefficients. $p$-value $<0.05$ was considered significant.

\section{Results}

Plasma ITLN1 protein levels increase while left ventricular ITLN1 RNA expression decreases following myocardial ischemia in humans

We used our prior CPB model in patients undergoing aortic valve replacement to obtain pre-ischemia (blood and left ventricular tissue harvested at the start of $\mathrm{CPB}$ ) and post-ischemia samples (tissue harvested after a mean of $81.73 \pm 27.89 \mathrm{~min}$ of ischemic cross clamp time) [21]. This model has previously been validated to mimic ischemia in human hearts [32, 33]. In a small cohort of patients, ITLN1 was the most significantly down-regulated gene in this model [21]. In the current larger study from 132 paired samples, median levels of pre-ischemia and postischemia left ventricular ITLN1 RNA expression were 59.96 FPKM (interquartile range 5.43-303.57) and 2.46 FPKM (interquartile range 0.84-11.00) respectively. In this population, post-ischemia RNA levels were significantly lower than pre-ischemia levels (mean difference 280.48, 95\% CI 449.43-111.54, $p=0.001$ ) (Fig. 1a). From the same group of patients, 106 paired samples had mean

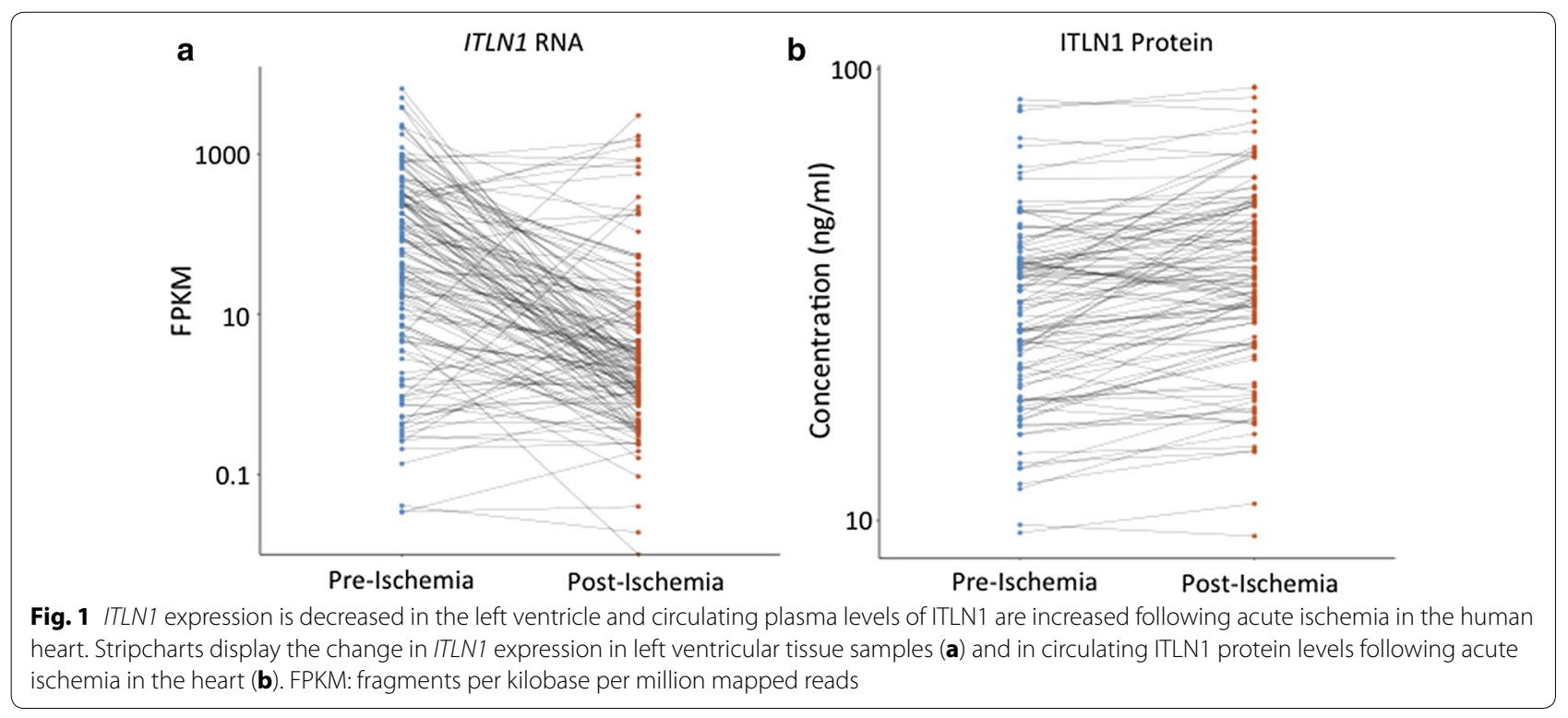


pre-ischemia and post-ischemia ITLN1 plasma levels of $32.23 \pm 15.22$ and $37.46 \pm 16.84 \mathrm{ng} / \mathrm{ml}$ respectively. In this population post-ischemia protein levels were significantly higher than pre-ischemia levels (mean difference 5.24, 95\% CI $3.52-6.95, p<0.001$ ) (Fig. 1b). In patients with CAD compared to those without, pre-ischemia left ventricular expression levels of ITLN1 were slightly higher (median 89.64 FPKM interquartile range $4.60-331.00$ vs median 43.53 interquartile range $5.62-281.85, p=0.839$ ) while pre-ischemia plasma levels of ITLN1 were slightly lower (mean $30.94 \pm 14.44 \mathrm{ng} / \mathrm{ml}$ vs mean $33.56 \pm 16.03 \mathrm{ng} / \mathrm{ml}$, $p=0.378$ ) but these associations were not significant.

Table 2 Univariate correlations between ITLN1 RNA expression and circulating levels of ITLN1 protein with clinical parameters

\begin{tabular}{|c|c|c|}
\hline Characteristic & Beta & $p$-value \\
\hline \multicolumn{3}{|l|}{ Pre-ischemia ITLN1 RNA } \\
\hline Age & 1.586 & 0.81 \\
\hline BMI & -0.40 & 0.971 \\
\hline $\mathrm{HgA1C}$ & -1.05 & 0.99 \\
\hline Sex (male) & -18.42 & 0.91 \\
\hline CAD & 32.15 & 0.839 \\
\hline Day 1 CKMB & 1.80 & 0.582 \\
\hline ITLN1 pre-ischemia protein & -1.06 & 0.879 \\
\hline \multicolumn{3}{|l|}{ Post-ischemia ITLN1 RNA } \\
\hline Age & 1.08 & 0.683 \\
\hline BMI & -0.43 & 0.923 \\
\hline $\mathrm{HgA1C}$ & -29.44 & 0.404 \\
\hline Sex (male) & -4.55 & 0.944 \\
\hline CAD & -2.97 & 0.962 \\
\hline Day 1 CKMB & -0.39 & 0.756 \\
\hline AX time & -0.50 & 0.660 \\
\hline \multicolumn{3}{|l|}{ Pre-ischemia ITLN1 protein } \\
\hline Age & 0.30 & $0.022^{*}$ \\
\hline BMI & -0.20 & 0.340 \\
\hline $\mathrm{HgA1c}$ & 2.18 & 0.225 \\
\hline Sex (male) & 3.37 & 0.260 \\
\hline CAD & -2.36 & 0.424 \\
\hline Day 1 CKMB & -0.01 & 0.900 \\
\hline ITLN1 pre-ischemia RNA & -0.0002 & 0.879 \\
\hline \multicolumn{3}{|l|}{ Post-ischemia ITLN1 protein } \\
\hline Age & 0.37 & $0.011^{*}$ \\
\hline BMI & -0.26 & 0.250 \\
\hline $\mathrm{HgA1C}$ & 1.64 & 0.410 \\
\hline Sex (male) & -1.00 & 0.765 \\
\hline CAD & -0.24 & 0.942 \\
\hline Day 1 CKMB & -0.04 & 0.524 \\
\hline AX time & -0.01 & 0.862 \\
\hline
\end{tabular}

$B M I$ body mass index, $\mathrm{HgAl} \mathrm{C}$ hemoglobin $\mathrm{A} 1 \mathrm{C}, \mathrm{CAD}$ coronary artery disease, $C K M B$ creatine kinase $M B$ fraction, $A X$ time aortic cross clamp time

* Denotes significance
We next investigated the correlation between preischemia and post-ischemia ITLN1 expression in the left ventricle and clinical parameters using univariate linear regression models. There were no significant correlations identified. We repeated these correlations with plasma protein levels. Age was positively correlated with plasma protein levels before and after ischemia (Table 2). There were no significant associations of plasma protein levels or left ventricular expression of ITLN1 with patients on or off statin medications (data not shown).

\section{Epicardial and visceral adipose tissue have a similar gene expression profile, characterized by enrichment of genes encoding proteins involved in innate immunity}

Given the proximity of our cardiac biopsy samples to epicardial adipose tissue (EAT), which has also been shown to express ITLN1 [8, 19], we further wanted to characterize the expression of ITLN1 in this compartment. Clinical studies have demonstrated that the amount of EAT correlates with visceral adipose tissue (VAT), and is increased in aging and obesity $[34,35]$. Therefore we hypothesized that the transcriptome of EAT may be similar to that of VAT, including high expression of ITLN1. In order to compare the gene expression profile of VAT with EAT, we utilized our previously published work [29]. We first compared gene expression in paired SAT and VAT samples from eight non-diabetic patients who underwent weight reduction surgery. Next, we compared gene expression in paired EAT and SAT samples from nine patients without CAD having surgery for heart disease. We then evaluated the two sets of differentially regulated genes in order to indirectly compare the gene expression of EAT and VAT. In the paired VAT vs. SAT comparison, there was differential expression of 845 genes (Fold change $>2.0, p<0.05$ ). In the paired SAT vs. EAT comparison, 188 genes were differentially expressed (FC $>2.0, p<0.05$ ). Of these 188 genes, $46 \%$ were commonly differentially regulated in VAT (Fig. 2). These included ITLN1 and other canonical VAT specific genes such as TCF21, SRFP2, and ALOX15 (Table 3). Remarkably, geneset enrichment analysis of the differentially expressed genes in both the SAT vs. VAT and SAT vs. EAT comparison yielded almost identical results (Table 4). The top three enriched pathways in VAT and EAT included complement, classical complement, and lectin induced complement. Therefore, even in the absence of CAD or diabetes, both VAT and EAT seem to play an important role in innate immunity.

\section{ITLN1 expression is localized to the mesothelium and downregulated following epithelial to mesenchymal transition}

Next, we aimed to more carefully characterize the expression of ITLN1 in EAT. We first confirmed that 


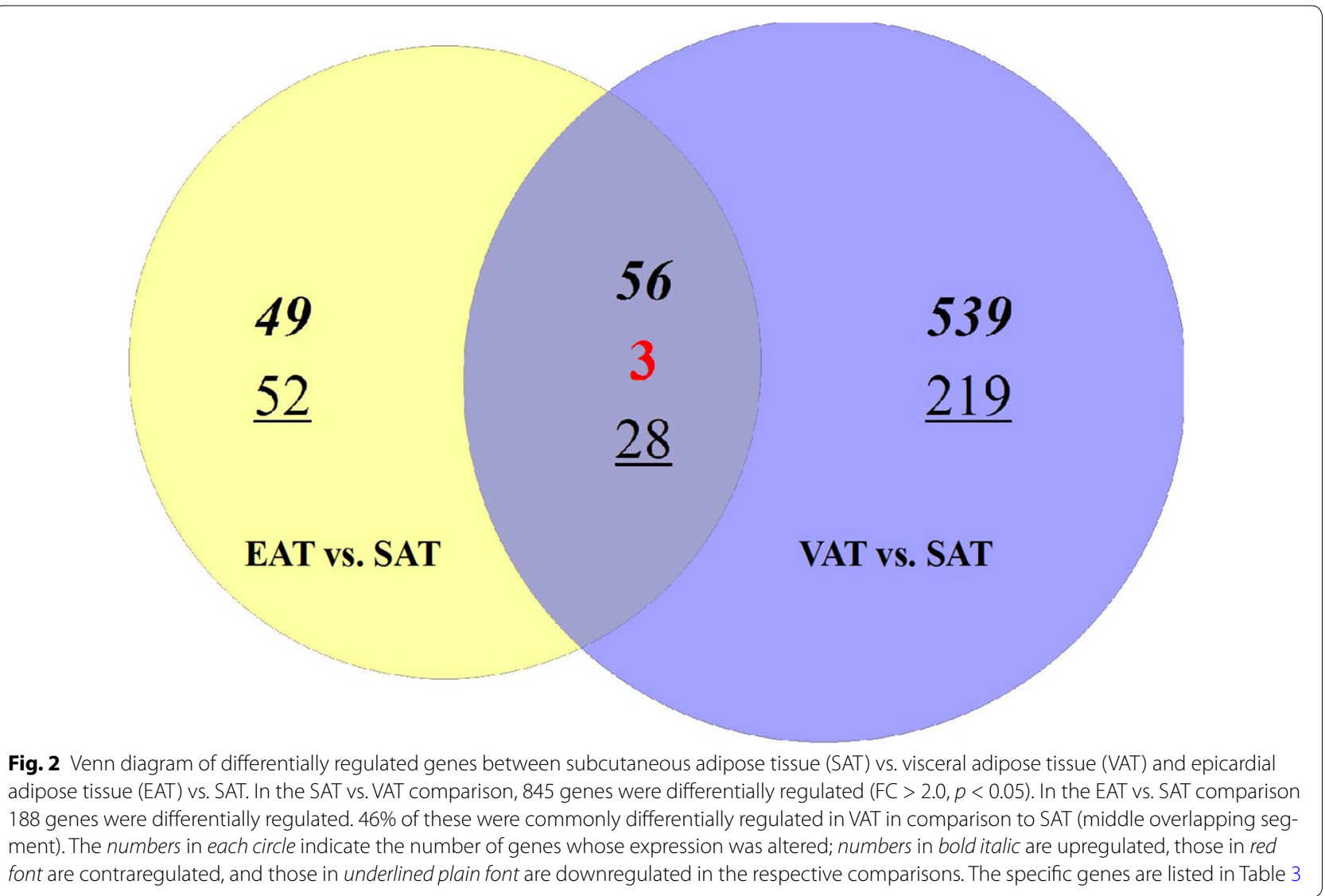

expression of ITLN1 is highly enriched in human VAT, and nearly absent in SAT (Fig. 3a). Similar to other studies $[7,8]$, we also were able to show that ITLN1 expression in human VAT is isolated to the stromal vascular fraction (SVF) (Fig. 3b). Mice, on the other hand, have equivalent low levels of ITLN1 expression in both VAT and SAT (Fig. 3c).

Since the SVF can contain multiple different cell types including epithelial cells, macrophages, fibroblasts, and precursory adipocytes [7], we sought to further characterize the location of ITLN1 in EAT. Immunohistochemistry demonstrated that ITLN1 is predominantly localized to the mesothelium, which constitutes the visceral pericardium, and not the adjacent adipocytes (Fig. 4a-d).

We then wanted to determine if ITLN1 expression requires an epithelial cell fate. Primary human mesothelial cells were treated with TGF $\beta 1(5 \mathrm{ng} / \mathrm{ml})$ for $48 \mathrm{~h}$ in order to induce epithelial to mesenchymal transition. qRT-PCR demonstrated that ITLN1 RNA expression was dramatically reduced within $48 \mathrm{~h}$ (Fig. $5 \mathrm{a}$ ). We also confirmed that expression of ITLN1 was absent in a human adipocyte cell line before and after adipogenic differentiation (SGBS D0 and D14); whereas the adipocyte specific gene Perilipin 1 (PLIN1) was increased dramatically in this cell line, but not in the mesothelial cells (Fig. 5b). Furthermore, using qRT-PCR, we confirmed that the decreased ITLN1 mRNA expression was paralleled by an early transition to a mesenchymal lineage, characterized by increased expression of the mesenchymal markers $V M A C$, SNAI1, and SNAI2 (Fig. 5c). Interestingly, many genes that have been shown to play a role in the epithelial to mesenchymal transition were among the complete list of RNA-seq differentially expressed genes from our pre- and post-ischemic human myocardial samples. In particular, there was upregulation of the mesenchymal markers MMP9, TCF4, BMP1, and SNAI2, and downregulation of the epithelial markers $K R T 19$ and $C D H-1$ (Additional file 1: Table S1).

\section{Discussion}

This is the first study to demonstrate changes in ITLN1 expression in a large cohort of human left ventricular tissue samples following acute ischemia. We show that pre-ischemia levels of ITLN1 expression in the human myocardium are much higher than the nominal levels depicted in the GTEX consortium [36]. This is likely a result of the tissue samples being immediately placed 
Table 3 Genes whose expression are commonly regulated in visceral adipose tissue (VAT) and epicardial adipose tissue (EAT) in comparison to subcutaneous adipose tissue (SAT)

\begin{tabular}{|c|c|c|}
\hline Commonly up regulated in VAT and EAT $(n=56)$ & $\begin{array}{l}\text { Contra regulated in VAT } \\
\text { and EAT }(n=3)\end{array}$ & $\begin{array}{l}\text { Commonly down regulated in VAT and EAT } \\
(n=28)\end{array}$ \\
\hline $\begin{array}{l}\text { ITLN1,TGM1, PROX1, IL18, C3, FRAS1, PDPN, PLAT, UPK3B, PDE1A, } \\
\text { CGNL1, OSR1, GATA6, KCNT2, C210rf62, FBLN1, DSC3, TFF3, RELN, } \\
\text { SULF1, CFI, FLRT3, PLLP, SLC39A8, GLT8D4, BCHE, MEIS2, PTPRD, } \\
\text { ALDH1A2, HSD17B6, GPM6A, ART4, TIMP1, DKFZP586H2123, SYT4, } \\
\text { BNC1, MSLN, PTGDS, LRP2, TGM2, ITGB8, PTN, ALOX15, C4A/C4B, } \\
\text { CLDN1, RARRES1, INMT, MMRN1, UPK1B, SLPI, C7, TCF21, CKMT1A, } \\
\text { CCL21, HP, PKHD1L1, }\end{array}$ & SFRP2, CD36, CHI3L2 & $\begin{array}{l}\text { NNAT, C12orf39, CXCL14, CRHBP, XG, ALDOC, ABCD2, } \\
\text { EGFL6, ADRA2A, MFAP5, CPM, APOB, SIM1, SIX1, } \\
\text { NOVA1, TBX15, SNX10, ENPP1, MME, OSR2, CDKN2B, } \\
\text { DGAT2, FOSB, CCND1, TNN, TBX5, ZFP36, KLB }\end{array}$ \\
\hline
\end{tabular}

in preservative unlike other samples exposed to longer periods of inadvertent ischemic time prior to harvesting. The ischemic insult of $\mathrm{CPB}$ led to a dramatic decrease in ITLN1 reads to very low detectable levels in most patients. Given results from prior studies which have shown a cardioprotective function of ITLN1 [5, 17], this decrease in endogenous ITLN1 RNA may make the heart vulnerable to further injury. There was also a negative correlation between aortic cross clamp time and the level of post-ischemia ITLN1 mRNA, although with the lack of significance we cannot conclude with certainty that longer ischemic time leads to lower levels of ITLN1 mRNA in the myocardium.

Prior studies have shown that plasma levels of ITLN1 are low in patients with CAD $[12,19]$. The plasma levels of ITLN1 prior to acute ischemia in our study were also lower in patients with known CAD, although this difference was not statistically significant. The response to acute ischemia has been less studied. One group showed that patients with acute coronary syndrome (ACS) have lower levels of plasma ITLN1 than healthy controls [13]. Our study using a large number of paired tissue samples, on the other hand, demonstrated an increase in plasma ITLN1 levels. This difference was significant regardless of underlying CAD. Those patients in the prior study with ACS likely had chronic levels of ischemia prior to presentation, and that may have been the driving force behind lower levels of ITLN1. As a result, chronic levels of low grade ischemia, as present in patients with CAD, may lead to low levels of plasma ITLN1, but acute ischemia may cause an abrupt increase. Future studies are needed to determine the source of this increased pool of circulating ITLN1. It could originate from the myocardium, which combined with lower levels of RNA expression would expose this organ to even more injury, or it could come from a visceral fat compartment in the body in order to rescue low myocardial expression. We did not identify a correlation between circulating levels of pre-ischemia ITLN1 protein and the myocardial injury marker CKMB on post-operative day 1. Nevertheless, the low variance in CKMB levels in our study does not refute other studies that have shown a potential protective effect of ITLN1 towards myocardial ischemia.

In agreement with previous work, we show that ITLN1 is also expressed in EAT and localized to the SVF [7, 8, 37]. We further expand this knowledge by showing that ITLN1 is confined to the mesothelial cells of the visceral pericardium or epicardium in human tissue through immunohistochemistry. Recent studies have shown that the mesothelial layer plays critical roles in development and disease processes in fat tissue in part due to its ability to secrete regulatory substances in a paracrine fashion [38]. This localization is additionally supported by evidence that ITLN1 expression is dependent on an epithelial cell fate as expression levels decrease following an epithelial to mesenchymal transformation. Interestingly, ischemic injury has been shown to stimulate an epithelial to mesenchymal transition in the epicardium, a process that can donate cells of multiple lineages to the myocardium [39, 40]. Many genes known to play a role in the epithelial to mesenchymal transition were dysregulated in our myocardial samples following ischemia. As a result, although it is possible that the changes in ITLN1 expression may come from myocytes in our biopsies, it is also possible that these changes occur in a population of cells that can undergo an epithelial to mesenchymal

Table 4 Gene set enrichment analysis of pathways enriched in epicardial adipose tissue (EAT) and visceral adipose tissue (VAT) vs. subcutaneous adipose tissue (SAT)

\begin{tabular}{lll}
\hline & Enrichment (\%) & $\boldsymbol{p}$-value \\
\hline Epicardial adipose tissue & & \\
Classical complement pathway & 28 & $<0.001$ \\
Lectin induced complement pathway & 25 & $<0.001$ \\
Complement pathway & 21 & $<0.001$ \\
Cell/molecules of local acute inflamma- & 17 & $<0.001$ \\
$\quad$ tory response & & \\
Visceral adipose tissue & 28 & $<0.001$ \\
Classical complement pathway & 26 & $<0.001$ \\
Complement pathway & 25 & $<0.001$ \\
\hline Lectin induced complement pathway & 25 \\
\hline
\end{tabular}



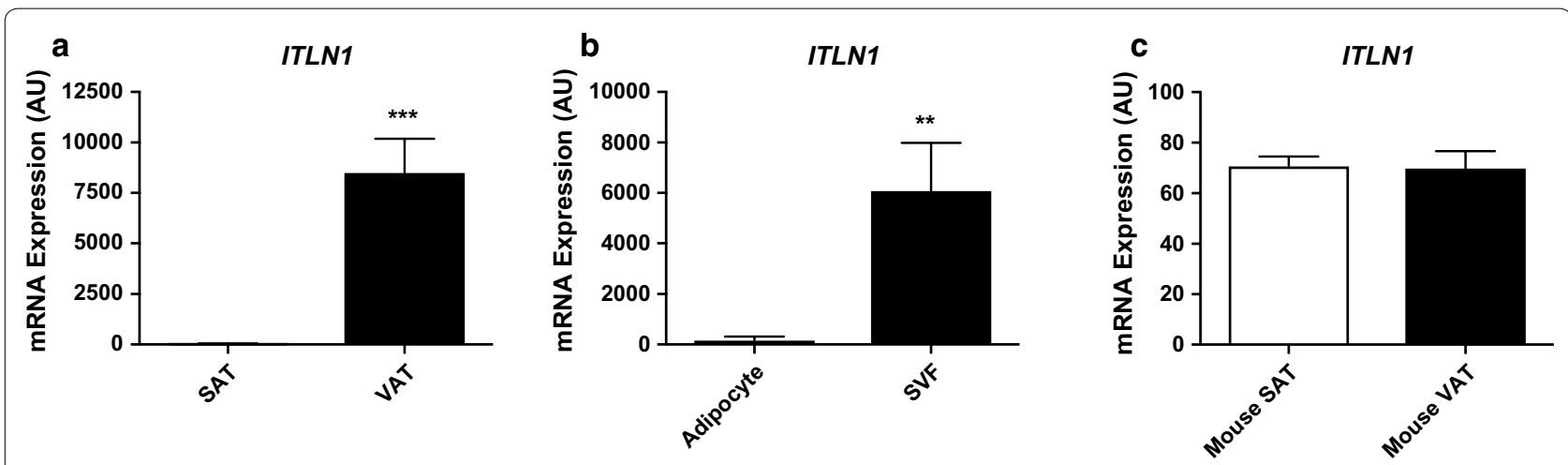

Fig. 3 ITLN1 is specifically expressed in the stromal vascular fraction of visceral fat in humans. a Microarrays were queried for expression of ITLN1 in visceral adipose tissue (VAT) vs. subcutaneous adipose tissue (SAT) in paired samples $\left.(n=8){ }^{* * *} p<0.001\right)$. b ITLN1 expression from microarrays of the adipocyte and stromal vascular fraction (SVF) of VAT in paired samples $\left.(n=6){ }^{* *} p<0.01\right)$. c Expression of ITLN1 from microarrays of murine SAT and VAT
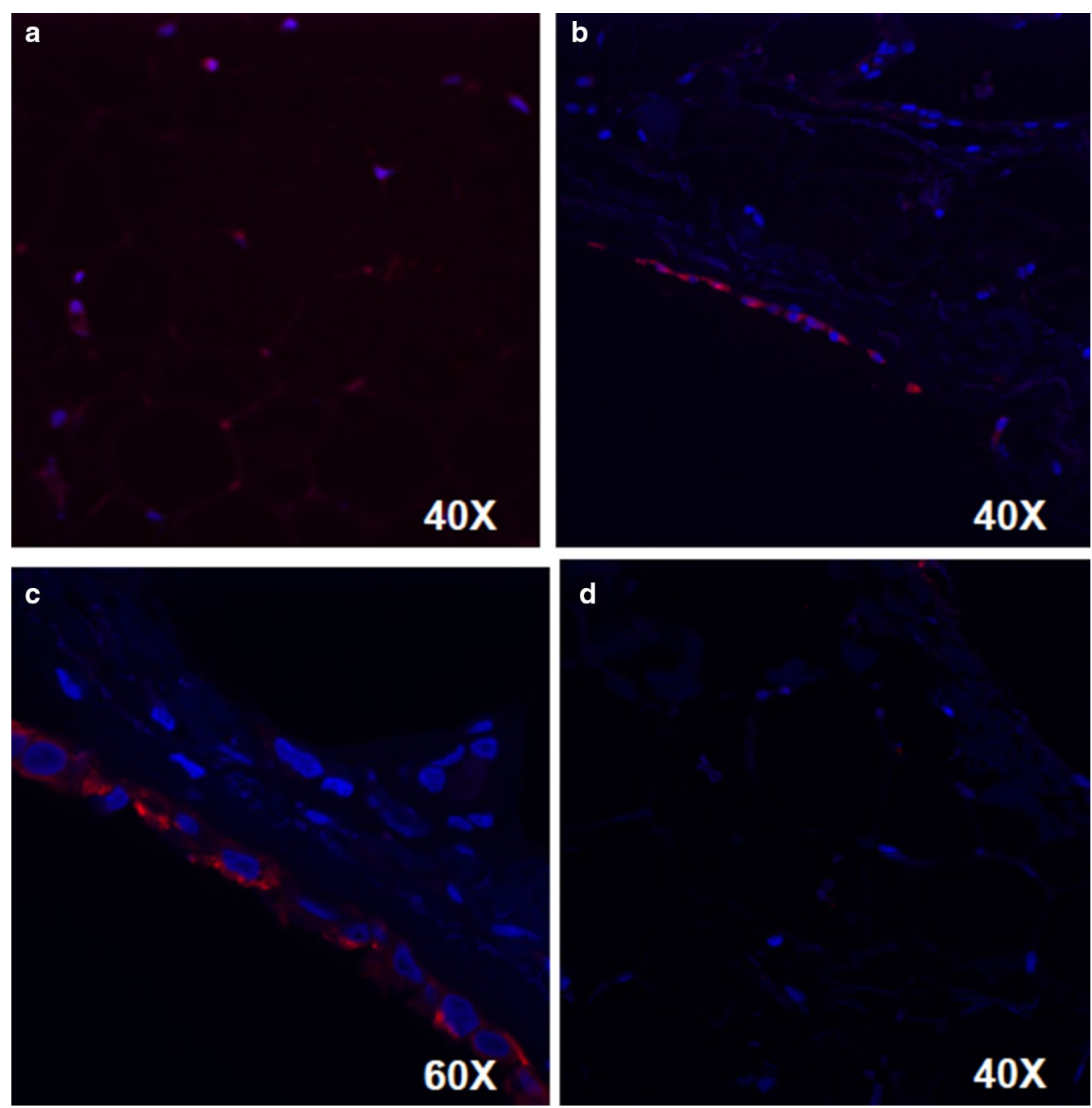

d

Fig. 4 Immunohistochemistry of ITLN1 in epicardial adipose tissue (EAT) shows enrichment within the mesothelial cell layer. Subcutaneous adipose tissue (SAT) stained for ITLN1 (a). EAT stained for ITLN1 (b) with magnification of the mesothelial cell layer of the visceral pericardium (c). Secondary antibody alone was used as a control (d). ITLN1 red, DAPI blue 

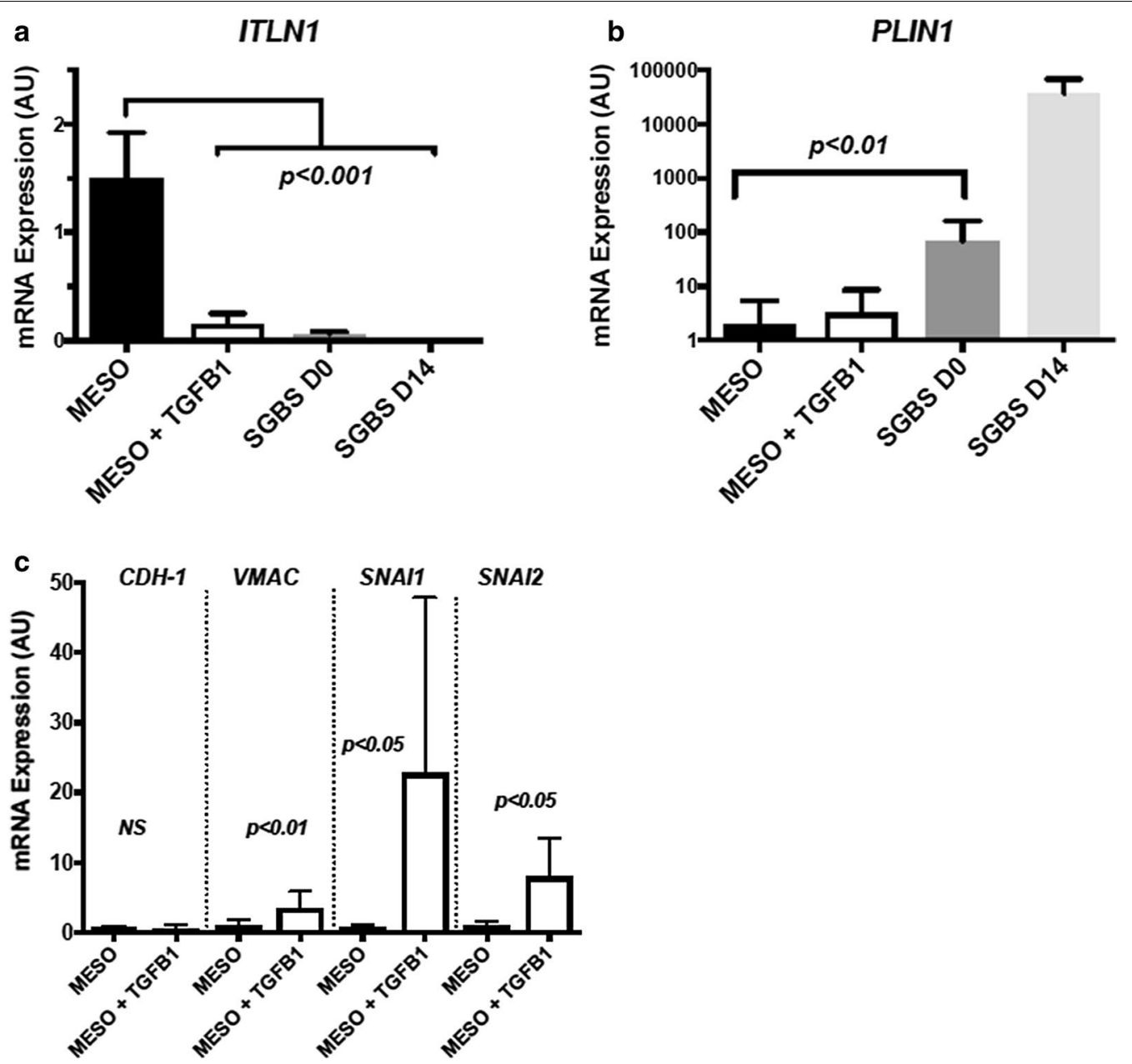

Fig. 5 ITLN1 expression in primary mesothelial cells is dramatically reduced following an epithelial to mesenchymal transition. a qRT-PCR for ITLN1 in mesothelial cells \pm TGF $\beta 1$ and in a human adipocyte cell line (SGBS) before (D0) and after differentiation (D14) ( $n=6$ per group). b qRT-PCR for the adipocyte specific gene PLIN1 in mesothelial cells \pm TGF $\beta 1$ and in a human adipocyte cell line (SGBS) before (D0) and after differentiation (D14) ( $n=6$ per group). $\mathbf{c}$ Expression of the epithelial cell marker $\mathrm{CDH}-1$ and the mesenchymal cell markers VMAC, SNAI1, and SNAI2 before and after treatment of mesothelial cells with TGF $\beta 1(n=6$ per group)

transition following injury such as the vascular endothelium, the endocardium, the epicardium, or cells in transition from the endocardium/epicardium to the myocardium. Additional studies are required to further define this cell population.

\section{Conclusions}

This is the first study to demonstrate an acute decline in left ventricular ITLN1 expression following ischemia in a large cohort of surgical patients. This is paralleled by an increase in plasma protein levels. We also provide evidence to suggest that the expression of ITLN1, similar to other secreted factors in the heart, may be dependent on an epithelial cell fate. Future studies will be needed to validate the precise sources and targets of
ITLN1 in the heart in order to further define its protective capabilities.

\section{Additional file}

Additional file 1: Table S1. Differentially expressed genes affiliated with the epithelial to mesenchymal transition. The following table lists those genes associated with the epithelial to mesenchymal transition which are also among the list of significant differentially expressed genes following ischemia in human myocardial tissue biopsies with adjusted $p$-value $<0.1$ (Benjamini Hochberg method).

\section{Abbreviations}

ITLN1: Omentin/Intelectin-1; VAT: visceral adipose tissue; SAT: subcutaneous adipose tissue; EAT: epicardial adipose tissue; SVF: stromal vascular fraction; CAD: coronary artery disease; CPB: cardiopulmonary bypass; FPKM: fragments per kilobase per million mapped reads; BMI: body mass index; HgA1c: 
hemoglobin A1c; CKMB: creatine kinase MB fraction; ACS: acute coronary syndrome.

\section{Authors' contributions}

LS devising concept and experimental design, analyzing correlations of ITLN1 expression and plasma ITLN1 levels with ischemia and patient characteristics, statistical summary of patient demographics, writing manuscript; SN analyzing expression pattern of ITLN1 in various tissues, conducting microarrays on human adipose tissue; OG analyzing expression pattern of ITLN1 in various tissues, conducting microarrays on human adipose tissue; MC analyzing expression pattern of ITLN1 in various tissues, conducting microarrays on human adipose tissue; JG RNA extraction and library preparation; SS devising concept and experimental design, CS devising concept and experimental design, RNA-seq, JS devising concept and experimental design, RNA-seq, SA acquiring tissue from surgery; SB devising concept and experimental design; TF conducting experiments on immunohistochemistry of ITLN1, epithelial/ mesenchymal expression of ITLN1, microarrays of human adipose tissue, analyzing expression pattern of ITLN1 in various tissues, writing manuscript, JM devising concept and experimental design, analyzing the expression pattern of ITLN1 in human tissue, writing manuscript. All authors were responsible for editing and approving the final manuscript. All authors read and approved the final manuscript.

\section{Author details}

1 Department of Anesthesiology and Perioperative Medicine, University of California Los Angeles, Los Angeles, CA, USA. ${ }^{2}$ Program in Molecular Medicine, University of Massachusetts Medical Center, Worcester, MA, USA. ${ }^{3}$ UT Southwestern Medical Center, Dallas, TX, USA. ${ }^{4}$ Program in Molecular Medicine, University of Massachusetts Medical School, Worcester, MA, USA. ${ }^{5}$ Department of Genetics, Harvard Medical School, Boston, MA, USA. ${ }^{6}$ Department of Anesthesiology, Perioperative and Pain Medicine, Brigham and Women's Hospital, Harvard Medical School, CWN L1, 75 Francis Street, Boston, MA 02115, USA. ${ }^{7}$ Division of Cardiovascular Medicine, Brigham and Women's Hospital, Howard Hughes Medical Institute, Boston, MA 02115, USA. ${ }^{8}$ Division of Cardiac Surgery, Brigham and Women's Hospital, Harvard Medical School, Boston, MA, USA. ${ }^{9}$ Cardiovascular Division, Department of Medicine, University of Massachusetts Medical School, Worcester, MA, USA.

\section{Acknowledgements}

We acknowledge the outstanding contributory efforts of the CABG Genomics research staff: James Gosnell, RN; Kujtim Bodinaku, MD; Svetlana Gorbatov, $\mathrm{MPH}$. We thank all study subjects who participated in the TRANSCRIBE Program.

This work should be attributed to the Department of Anesthesiology, Perioperative and Pain Medicine, Brigham and Women's Hospital.

\section{Competing interests}

The authors declare that they have no competing interests.

\section{Availability of data and materials}

The human heart data is currently in submission at dbGap. The human adipose tissue can be found in the GEO database under accession code GSE28440.

\section{Ethics approval}

For human heart tissue, written informed consent was obtained from each patient and the study was approved by the Partners HealthCare Institutional Review Board. For human adipose tissue, written and informed consent was obtained from each patient and the study was approved by the University of Massachusetts Medical School Review Board.

\section{Funding}

The authors acknowledge support for this research by the National Institute of Health (Bethesda, MD) grant HL118266 (JDM) and R37DK030898 (MPC), the American Heart Association \#12FTF11260010 (TPF), The Harvard Catalyst, The Watkins Cardiovascular Discovery Award (Brigham and Women's Hospital, Boston, MA), the Scholars in Clinical Science Program (Harvard Medical School) and a Mercedes Concepcion Faculty Development Fellowship and Scholar in Translational Anesthesia Research grant from the Department of
Anesthesiology, Perioperative and Pain Medicine, (Brigham and Women's Hospital, Harvard Medical School, Boston, MA).

\section{Publisher's Note}

Springer Nature remains neutral with regard to jurisdictional claims in published maps and institutional affiliations.

Received: 10 May 2017 Accepted: 21 June 2017

Published online: 07 July 2017

\section{References}

1. Ouchi N, Parker JL, Lugus JJ, Walsh K. Adipokines in inflammation and metabolic disease. Nat Rev Immunol. 2011;11(2):85-97.

2. Rabe K, Lehrke M, Parhofer KG, BroedI UC. Adipokines and insulin resistance. Mol Med. 2008;14(11-12):741-51.

3. Rega-Kaun G, Kaun C, Wojta J. More than a simple storage organ: adipose tissue as a source of adipokines involved in cardiovascular disease. Thromb Haemost. 2013;110(4):641-50.

4. Katsi V, Vamvakou G, Lekakis J, Tousoulis D, Stefanadis C, Makris T, Kallikazaros I. Omentin, fat and heart: classical music with new instruments. Heart Lung Circ. 2014;23(9):802-6.

5. Mattu HS, Randeva HS. Role of adipokines in cardiovascular disease. J Endocrinol. 2013;216(1):T17-36.

6. Shibata R, Ohashi K, Murohara T, Ouchi N. The potential of adipokines as therapeutic agents for cardiovascular disease. Cytokine Growth Factor Rev. 2014;25(4):483-7

7. Yang R-Z, Lee M-J, Hu H, Pray J, Wu H-B, Hansen BC, Shuldiner AR, Fried SK, McLenithan JC, Gong D-W. Identification of omentin as a novel depotspecific adipokine in human adipose tissue: possible role in modulating insulin action. Am J Physiol-Endocrinol Metab. 2006;290(6):E1253.

8. Fain JN, Sacks HS, Buehrer B, Bahouth SW, Garrett E, Wolf RY, Carter RA, Tichansky DS, Madan AK. Identification of omentin mRNA in human epicardial adipose tissue: comparison to omentin in subcutaneous, internal mammary artery periadventitial and visceral abdominal depots. Int J Obes (Lond). 2008;32(5):810-5.

9. Pan HY, Guo L, Li Q. Changes of serum omentin-1 levels in normal subjects and in patients with impaired glucose regulation and with newly diagnosed and untreated type 2 diabetes. Diabetes Res Clin Pract. 2010;88(1):29-33.

10. de Souza Batista CM, Yang RZ, Lee MJ, Glynn NM, Yu DZ, Pray J, Ndubuizu K, Patil S, Schwartz A, Kligman M, et al. Omentin plasma levels and gene expression are decreased in obesity. Diabetes. 2007;56(6):1655-61.

11. Shibata R, Takahashi R, Kataoka Y, Ohashi K, Ikeda N, Kihara S, Murohara T, Ouchi N. Association of a fat-derived plasma protein omentin with carotid artery intima-media thickness in apparently healthy men. Hypertens Res. 2011;34(12):1309-12.

12. Shibata R, Ouchi N, Kikuchi R, Takahashi R, Takeshita K, Kataoka Y, Ohashi K, Ikeda N, Kihara S, Murohara T. Circulating omentin is associated with coronary artery disease in men. Atherosclerosis. 2011;219(2):811-4.

13. Zhong $X$, Zhang HY, Tan H, Zhou Y, Liu FL, Chen FQ, Shang DY. Association of serum omentin-1 levels with coronary artery disease. Acta Pharmacol Sin. 2011;32(7):873-8.

14. Yamawaki H, Kuramoto J, Kameshima S, Usui T, Okada M, Hara Y. Omentin, a novel adipocytokine inhibits TNF-induced vascular inflammation in human endothelial cells. Biochem Biophys Res Commun. 2011;408(2):339-43.

15. Yamawaki H, Tsubaki N, Mukohda M, Okada M, Hara Y. Omentin, a novel adipokine, induces vasodilation in rat isolated blood vessels. Biochem Biophys Res Commun. 2010;393(4):668-72.

16. Gu N, Dong Y, Tian Y, Di Z, Liu Z, Chang M, Jia X, Qian Y, Zhang W. Antiapoptotic and angiogenic effects of intelectin-1 in rat cerebral ischemia. Brain Res Bull. 2016;130:27-35.

17. Kataoka Y, Shibata R, Ohashi K, Kambara T, Enomoto T, Uemura Y, Ogura Y, Yuasa D, Matsuo K, Nagata T, et al. Omentin prevents myocardial ischemic injury through AMP-activated protein kinase- and Akt-dependent mechanisms. J Am Coll Cardiol. 2014;63(24):2722-33. 
18. Pourbehi MR, Zahedi T, Darabi H, Ostovar A, Assadi M, Nabipour I. Omentin-1 and nonfatal ischemic heart disease in postmenopausal women: a population-based study. Endocr Pract. 2016;22(7):780-5.

19. Harada K, Shibata R, Ouchi N, Tokuda Y, Funakubo H, Suzuki M, Kataoka T, Nagao T, Okumura S, Shinoda N, et al. Increased expression of the adipocytokine omentin in the epicardial adipose tissue of coronary artery disease patients. Atherosclerosis. 2016;251:299-304.

20. Du Y, Ji Q, Cai L, Huang F, Lai Y, Liu Y, Yu J, Han B, Zhu E, Zhang J, et al. Association between omentin-1 expression in human epicardial adipose tissue and coronary atherosclerosis. Cardiovasc Diabetol. 2016;15(1):90.

21. Muehlschlegel JD, Christodoulou DC, McKean D, Gorham J, Mazaika E, Heydarpour M, Lee G, DePalma SR, Perry TE, Fox AA, et al. Using nextgeneration RNA sequencing to examine ischemic changes induced by cold blood cardioplegia on the human left ventricular myocardium transcriptome. Anesthesiology. 2015;122(3):537-50.

22. Trapnell C, Pachter L, Salzberg SL. TopHat: discovering splice junctions with RNA-Seq. Bioinformatics (Oxford, England). 2009;25(9):1105-11.

23. Trapnell C, Roberts A, Goff L, Pertea G, Kim D, Kelley DR, Pimentel H, Salzberg SL, Rinn JL, Pachter L. Differential gene and transcript expression analysis of RNA-seq experiments with TopHat and Cufflinks. Nat Protoc. 2012;7(3):562-78.

24. Langmead B, Trapnell C, Pop M, Salzberg SL. Ultrafast and memoryefficient alignment of short DNA sequences to the human genome. Genome Biol. 2009;10(3):R25.

25. Trapnell C, Williams BA, Pertea G, Mortazavi A, Kwan G, van Baren MJ, Salzberg SL, Wold BJ, Pachter L. Transcript assembly and quantification by RNA-Seq reveals unannotated transcripts and isoform switching during cell differentiation. Nat Biotechnol. 2010;28(5):511-5.

26. Ritchie ME, Phipson B, Wu D, Hu Y, Law CW, Shi W, Smyth GK. limma powers differential expression analyses for RNA-sequencing and microarray studies. Nucleic Acids Res. 2015;43(7):e47.

27. Liu R, Holik AZ, Su S, Jansz N, Chen K, Leong HS, Blewitt ME, Asselin-Labat ML, Smyth GK, Ritchie ME. Why weight? Modelling sample and observational level variability improves power in RNA-seq analyses. Nucleic Acids Res. 2015;43(15):e97.

28. Robinson MD, McCarthy DJ, Smyth GK. edgeR: a Bioconductor package for differential expression analysis of digital gene expression data. Bioinformatics (Oxford, England). 2010;26(1):139-40.

29. Hardy OT, Perugini RA, Nicoloro SM, Gallagher-Dorval K, Puri V, Straubhaar J, Czech MP. Body mass index-independent inflammation in omental adipose tissue associated with insulin resistance in morbid obesity. Surg Obes Relat Dis. 2011;7(1):60-7.
30. Livak KJ, Schmittgen TD. Analysis of relative gene expression data using real-time quantitative PCR and the 2(-Delta Delta $C(T)$ ) method. Methods. 2001;25(4):402-8.

31. Team RC. R: A language and environment for statistical computing. Vienna: R Foundation for Statistical Computing; 2015

32. Muehlschlegel JD, Perry TE, Liu KY, Nascimben L, Fox AA, Collard CD, Avery EG, Aranki SF, D'Ambra MN, Shernan SK, et al. Troponin is superior to electrocardiogram and creatinine kinase MB for predicting clinically significant myocardial injury after coronary artery bypass grafting. Eur Heart J. 2009;30(13):1574-83.

33. Malmberg M, Parkka J, Vahasilta T, Saraste A, Laitio T, Kiss J, Latva-Hirvela J, Saukko P, Savunen T. Cardiomyocyte apoptosis after cardioplegic ischemia: comparison to unprotected regional ischemia-reperfusion. Eur Surg Res. 2011;46(1):19-25.

34. Fitzgibbons TP, Czech MP. Epicardial and perivascular adipose tissues and their influence on cardiovascular disease: basic mechanisms and clinical associations. J Am Heart Assoc. 2014;3(2):e000582.

35. lacobellis G, Assael F, Ribaudo MC, Zappaterreno A, Alessi G, Di Mario $U$, Leonetti F. Epicardial fat from echocardiography: a new method for visceral adipose tissue prediction. Obes Res. 2003;11(2):304-10.

36. Lonsdale J, Thomas J, Salvatore M, Phillips R, Lo E, Shad S, Hasz R, Walters G, Garcia F, Young N, et al. The genotype-tissue expression (GTEx) project. Nat Genet. 2013;45(6):580-5.

37. Gaborit B, Venteclef N, Ancel P, Pelloux V, Gariboldi V, Leprince P, Amour J, Hatem SN, Jouve E, Dutour A, et al. Human epicardial adipose tissue has a specific transcriptomic signature depending on its anatomical peri-atrial, peri-ventricular, or peri-coronary location. Cardiovasc Res. 2015;108(1):62-73.

38. Gupta OT, Gupta RK. Visceral adipose tissue mesothelial cells: living on the edge or just taking up space? Trends Endocrinol Metab: TEM. 2015;26(10):515-23.

39. Krainock M, Toubat O, Danopoulos S, Beckham A, Warburton D, Kim R. Epicardial epithelial-to-mesenchymal transition in heart development and disease. J Clin Med. 2016;5(2):27.

40. Zhou B, Pu WT. Epicardial epithelial-to-mesenchymal transition in injured heart. J Cell Mol Med. 2011;15(12):2781-3.

\section{Submit your next manuscript to BioMed Central and we will help you at every step:}

- We accept pre-submission inquiries

- Our selector tool helps you to find the most relevant journal

- We provide round the clock customer support

- Convenient online submission

- Thorough peer review

- Inclusion in PubMed and all major indexing services

- Maximum visibility for your research

Submit your manuscript at www.biomedcentral.com/submit
() Biomed Central 